\title{
Construir la Historia a través de las competencias educativas en la enseñanza universitaria
}

\section{Build History through educational competencies in university education}

\author{
María Pilar Molina Torres ${ }^{1}$, Ana María Niveau de Villedary y Mariñas $^{2} \&$ \\ Raimundo Fco. Ortiz Urbano ${ }^{3}$
}

Fecha de recepción: 18/11/2019; Fecha de revisión: 30/11/2019; Fecha de aceptación: 25/02/2020

Cómo citar este artículo:

Molina, M. P, Niveau, A \& Ortiz, R. (2020). Construir la Historia a través de las competencias educativas en la enseñanza universitaria. Revista de Innovación y Buenas Prácticas Docentes, 9(1), 13-23.

\section{Autor de Correspondencia: pilar.molina@uco.es}

\begin{abstract}
Resumen:
Con este trabajo se implementaron las distintas competencias educativas presentes en los proyectos curriculares relacionados con el área de Didáctica de las Ciencias Sociales y de la Historia. Como objetivo principal nos propusimos contextualizar al alumnado de tercer curso del Grado de Educación Primaria con la elaboración de una serie de talleres didácticos y con ello contribuir a la preservación y valoración que el Patrimonio Histórico tiene en nuestra realidad social. Mediante una metodología investigativa se llevarán a cabo varias tareas prácticas, como pequeños trabajos de investigación, la realización de maquetas históricas referentes al tema elegido en el itinerario didáctico que volcarán en un blog de aula grupal y una visita al Museo Arqueológico de Córdoba. Los resultados se centraron en la interrelación de dos ramas diferentes de conocimiento (Ciencias Sociales y Humanidades), lo que nos permitió trabajar de manera interdisciplinar para concretar en la práctica las diferentes actividades y adentrarnos en una experiencia real de Educación Patrimonial. En conclusión, la finalidad de esta experiencia didáctica es que los estudiantes puedan aplicarlas y llevarlas a un escenario práctico de innovación educacional.
\end{abstract}

Palabras clave: historia, innovación, metodología, patrimonio.

\begin{abstract}
:
With this work the different educational competences present in the curricular projects related to the area of Didactics of Social Sciences and History were implemented. As a main objective we set out to contextualize the students of the third year of the Primary Education Degree with the elaboration of a series of didactic workshops and with this contribute to the preservation and valuation that the Historical Heritage has in our social reality. Through a research methodology several practical tasks will be carried out, such as small research projects, the realization of historical models referring to the theme chosen in the didactic itinerary that will be turned into a group classroom blog and a visit to the Archaeological Museum of Córdoba. The results focused on the interrelation of two different branches of knowledge (Social Sciences and Humanities), which allowed us to work in an interdisciplinary way to concretize the different activities in practice and enter a real experience of Heritage Education. In conclusion, the purpose of this teaching experience is that students can apply them and take them to a practical scenario of educational innovation.
\end{abstract}

Key Words: history, innovation, methodology, heritage.

\footnotetext{
1 Universidad de Córdoba (España), pilar.molina@uco.es; CÓDIGO ORCID: http://orcid.org/0000-00017157-0324

2 Universidad de Cádiz (España), anamaria.niveau@uca.es; CÓDIGO ORCID: http://orcid.org/0000-00028888-1169

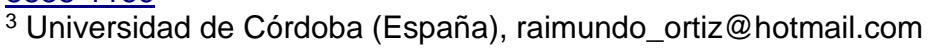




\section{INTRODUCCIÓN}

El desarrollo de esta experiencia "Construir la Historia a través de las competencias educativas en el Grado de Educación Primaria" nació de manera natural sin forzar la motivación y el interés que podía suscitar la temática centrada en la Educación Patrimonial. Para ello, y desde el primer momento, se familiarizó al alumnado con el estilo de vida que se desarrolló en una ciudad romana, con la finalidad de promover un acercamiento al patrimonio histórico y arqueológico (Ballart, 2002). Asimismo, desde el punto de vista de la educación patrimonial, se debe puntualizar que la definición de patrimonio cultural ha cambiado en los últimos años considerablemente, quedando ligada a los conceptos de cultura y sociedad (Hernández y Guillén, 2017).

La experiencia didáctica se desarrolló mediante una metodología por proyectos que fomentó el aprendizaje cooperativo y la elaboración en el aula de diferentes actividades prácticas, que se completaron con un itinerario realizado por los/as alumnos/as y con una visita al Museo Arqueológico de Córdoba. Para las diferentes fases del proyecto y la secuencia de actividades, los/as estudiantes decidieron la manera más adecuada de elaborar una propuesta que sirviera, por un lado, para completar los conocimientos ya adquiridos a través de sus experiencias personales y, por otro lado, para mejorar su aprendizaje profesional (Moliner \& Sánchez-Tarazaga, 2015; Navarro, González \& Botella, 2015). En este contexto, Kolmos (2004, p.85) afirma que: "El trabajo por proyectos se caracteriza por ser un trabajo que es fruto de la elaboración grupal". Es aquí donde la literatura científica nos aporta otras opiniones acerca del aprendizaje de las Ciencias Sociales. Así pues, como puntualiza GarcíaValcárcel \& Basilotta (2017, p.115): "Es necesario que el proyecto esté centrado en el estudiante, adaptado a sus necesidades e intereses, despierte su curiosidad y genere motivación intrínseca". Es más, son los intereses preferentes de los/as alumnos/as universitarios/as los que fomentan el área de Ciencias Sociales y su implementación en el aula, al ser una materia que comparte líneas temáticas con el estudio del espacio geográfico y del tiempo histórico.

De hecho, el aprendizaje cooperativo y el desarrollo de las competencias educativas han fomentado la autonomía e iniciativa personal del alumnado del Grado de Educación Primaria. La interacción entre compañeros y la construcción de saberes propios proporcionan una serie de cuestiones reflexivas que involucraron a todo el grupo para conformar el pensamiento histórico y las narrativas personales (Rüsen, 2005; Palma, 2013; Saiz \& Gómez, 2016). Del mismo modo, el interés por la experimentación y la materia trabajada "La Corduba romana" ha generado una mayor capacidad para aprender a aprender y forjar el trabajo autónomo (Pérez, 2010). En definitiva, las tareas competenciales quedaron establecidas tanto por los/as estudiantes como por el profesorado de manera que como nos dicen Cuenca, Estepa y Martín (2011, p.46): "La didáctica del patrimonio no constituye un fin en sí mismo, sino que debe integrarse en el proceso educativo, dentro de las grandes metas establecidas para la educación reglada, particularmente en la formación de la ciudadanía". Es así como pudimos acercarnos e implicar al alumnado con los acontecimientos históricos del pasado y los conocimientos que suceden dentro y fuera de la universidad. En este sentido, acondicionamos un espacio (el aula) que contara con un elemento de referencia universitario, para orientar al alumnado en la experiencia formativa. Es necesario indicar que las decisiones grupales e individuales se adaptaron a las diversas estrategias educativas para conocer más en profundidad el patrimonio de la ciudad y, además, reforzar los contenidos curriculares. 
De este modo, las sinergias didácticas facilitan un proceso de enseñanza aprendizaje colaborativo e interactivo para enseñar y comprender la Historia (López, Miralles, Prats \& Gómez, 2017). A este respecto, la enseñanza de la Didáctica de la Historia es indispensable para afrontar en la práctica educativa los conocimientos patrimoniales que están incluidos en el desarrollo de las competencias (Perrenoud, 2004; Salinas, De Benito \& Lizana, 2014). De hecho, la educación patrimonial se presenta como un elemento educativo que debe ser adquirido por los docentes, y en nuestro caso concreto por los estudiantes del Grado de Educación Primaria (Molina, 2018a).

Ahora bien, para sensibilizar al alumnado en relación con los recursos patrimoniales de la etapa histórica estudiada, los objetivos que se plantearon y se cumplieron durante el proyecto se pueden dividir en dos dimensiones, teniendo como destinatarios a:

Por un lado, al alumnado:

- Tomar conciencia sobre la importancia que tiene la conservación del patrimonio.

- Desarrollar actitudes favorables hacia el trabajo colaborativo.

- Diseñar diferentes tipos de actividades dependiendo de los contenidos a tratar.

- Conocer y aplicar en las aulas las TIC.

Y, por otro lado, al profesorado:

- Fomentar en el alumnado el interés por el patrimonio cultural e histórico, en especial por nuestra ciudad.

- Reflexionar sobre las prácticas de aula para innovar y mejorar la labor docente.

- Alcanzar una mayor coordinación entre los equipos multidisciplinares implicados en el proyecto.

- Estimular la metodología investigativa desde la enseñanza universitaria.

\section{DESARROLLO DE LA EXPERIENCIA DE INNOVACIÓN}

Mediante el aprendizaje basado en proyectos pudimos adentrarnos en la Corduba romana, investigar sus prácticas cotidianas, integrarnos en la ordenación urbanística de la ciudad y proporcionar al alumnado un papel activo dentro del proceso de enseñanza y aprendizaje (Rekalde \& García, 2015; Vergara, 2016; Molina, 2018b). Así pues, la temática se abordó de forma innovadora al combinar de manera simultánea e interdisciplinar una serie de talleres didácticos y las TIC, concretamente un blog de aula (Cebrián, 2003; Durán, 2011). Los resultados obtenidos del proyecto han demostrado que el alumnado a través del trabajo cooperativo (Riera, 2011) y un aprendizaje significativo construye de manera autónoma sus propios conocimientos, y además les permitió concienciarlos de que la enseñanza del patrimonio cultural romano es parte esencial de nuestra cultura.

En este sentido, para alcanzar los objetivos expuestos se eliminaron las clases magistrales, optando por una metodología investigativa y con carácter constructivista, de manera que puedan aportar sus conocimientos y adquirir nuevas habilidades para acometer un aprendizaje significativo (Latorre, Rincón \& Arnal, 2005). Para ello, hemos contado con dos grupos de estudiantes, cada uno de sesenta alumnos y alumnas aproximadamente. La temporalización de la propuesta didáctica abarcó cinco semanas de sesiones prácticas, cuatro horas y media semanales, en la asignatura de Didáctica de las Ciencias Sociales en el Grado de Educación Primaria. 
Durante el proceso que se ha llevado a cabo en el curso 2017/2018, también el profesorado especialista en la Edad Antigua y Arqueología ayudó al profesorado dedicado a la parte Didáctica y complementó los recursos didácticos con las explicaciones adecuadas sobre contenidos históricos y arqueológicos de la ciudad en la Antigüedad. De este modo, la planificación inicial se configuró en torno a los conocimientos previos y a la contextualización histórica del alumnado. En principio, se les motivó para que expresaran qué sabían hacer y qué querían aprender. Así programamos un recorrido por seis temáticas que se trabajaron fuera mediante un itinerario y dentro del aula: (1) puerto fluvial, (2) actividades comerciales, (3) creencias religiosas, (4) teatro de la colonia, (5) espacio público y administrativo y, por último, (6) ludi romanos: anfiteatro.

Para elaborar este recurso planificamos un itinerario didáctico con Google Maps que plasmamos en un callejero actual de nuestra ciudad. A partir de este recurso, que no fue una simple visita guiada sino diseñada y elaborada por los/as estudiantes, pudimos trabajar las seis temáticas citadas y asimismo exponer lo que habían preparado en las paradas correspondientes. Divididos en pequeños equipos de investigación (4-5 alumnos/as), cada uno de ellos se encargó de recoger toda la información sobre las diferentes paradas que realizaron y que explicarán a sus compañeros como expertos en las materias detalladas (Figura 1).

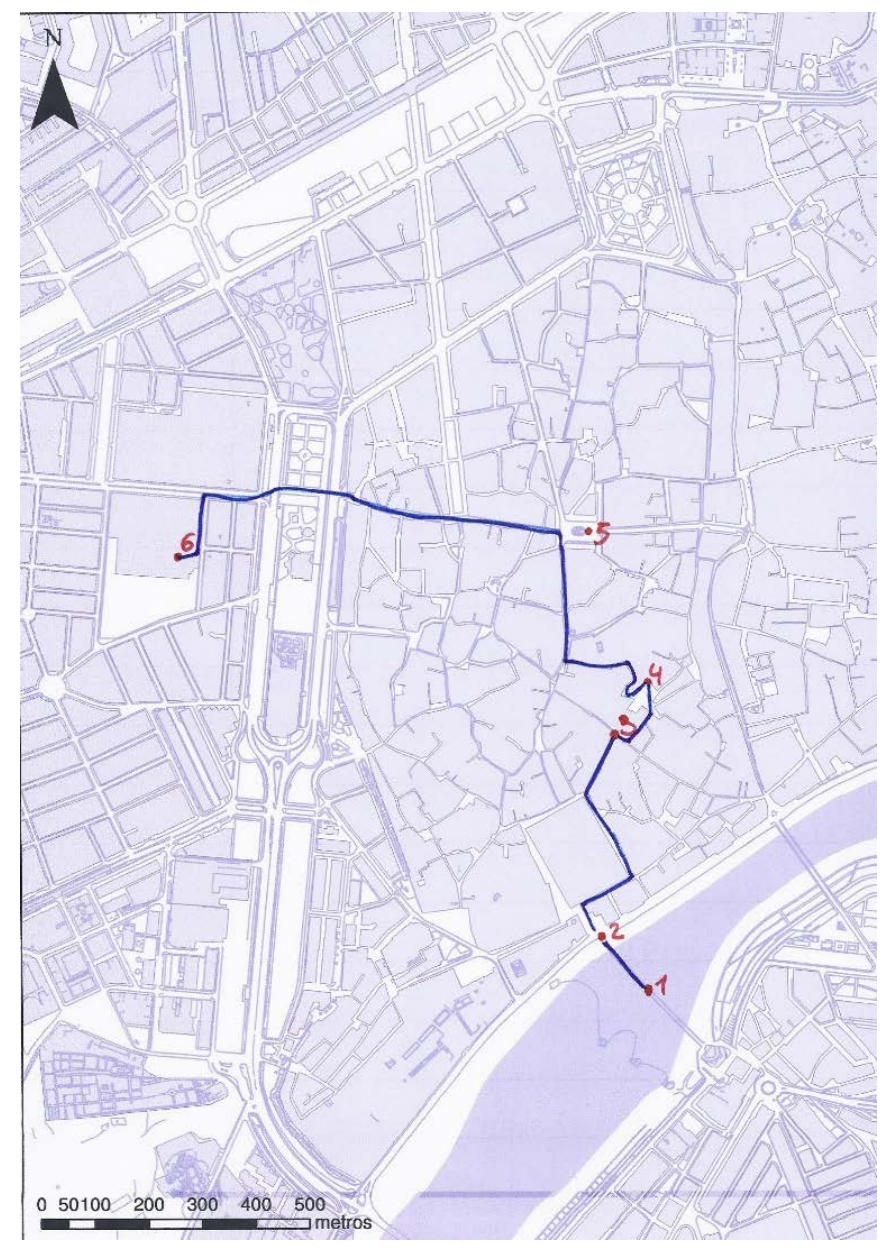

Figura 1. Recorrido didáctico diseñado por el alumnado sobre un callejero actual de Córdoba. 
En torno a la investigación, se plantearon tres cuestiones que trabajaron todos los grupos:

- ¿Qué función tenía este lugar en época romana?

- ¿Por qué se eligió este espacio para su construcción?

- ¿Cómo podrías explicar (con un recurso didáctico) tu parada a un grupo de alumnos/as de Educación Primaria?

Por lo que respecta a las paradas, el itinerario siguió el orden que se recoge:

1.- Puente romano (Puerto romano)

2.- Puerta del puente (Economía y comercio)

3.- Altos de Santa Ana (Espacio religioso de culto público)

4.- Teatro romano (Restos in situ del edificio en el Museo Arqueológico de Córdoba)

5.- Plaza de las Tendillas (Foro romano y administración municipal)

6.- Rectorado de la Universidad de Córdoba (Anfiteatro, ludi romanos)

De esta manera, las investigaciones previas que organizamos antes de la salida y que dieron sentido a las explicaciones en dichas paradas, se volcaron en un blog de aula que utilizaron para compartir y actualizar las actividades de carácter experiencial que afianzaron la práctica y la teoría en las exposiciones. Con el fin de que dieran forma a lo aprendido y explicado en clase, se pidió a los/as alumnos/as que reflexionaran sobre el enfoque didáctico que darían a las cuestiones formuladas y elaboraran su propia propuesta. Los materiales empleados en clase y la bibliografía sobre el tema se colgaron en Moodle para que pudieran disponer de estos recursos sobre la Corduba romana (mapas interactivos, webquest, vídeos didácticos bilingües como por ejemplo el Crash Course World History, puzles virtuales de conceptos, etc.) (Figuras 2 y 3 ).

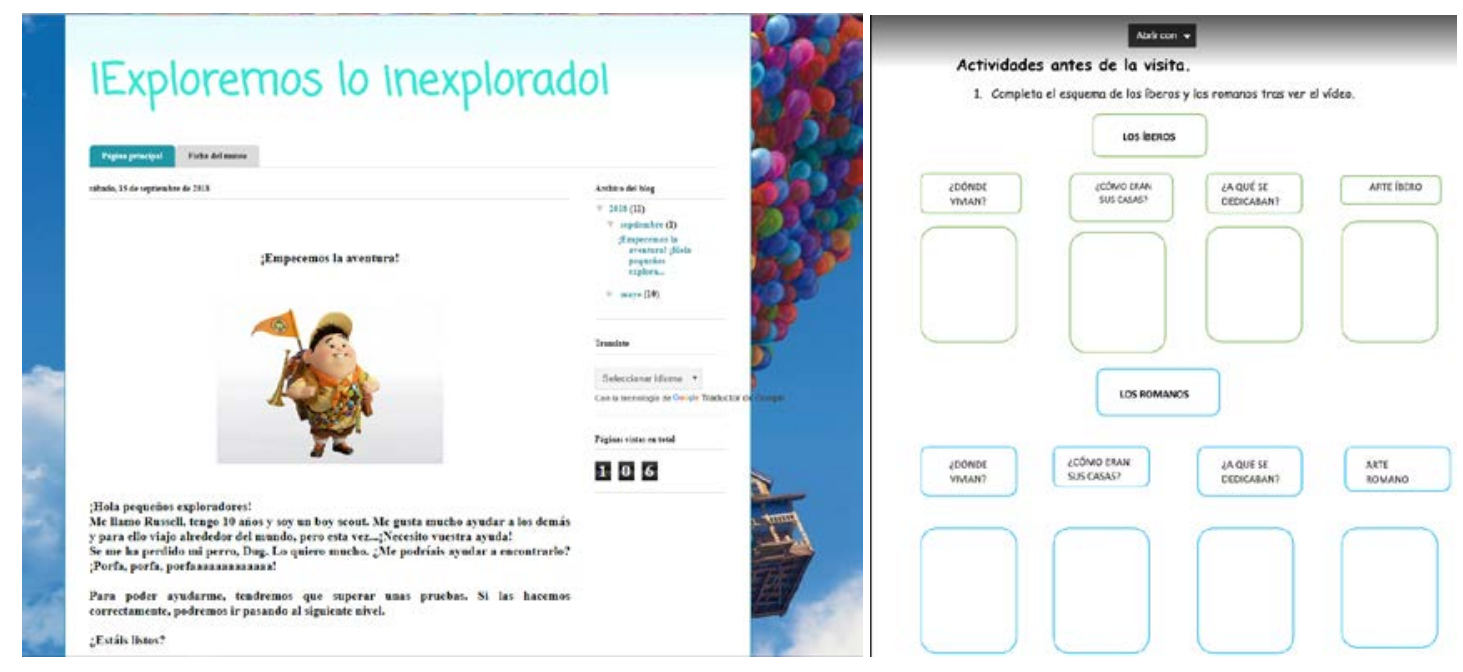

Figuras 2-3. Captura de pantalla del blog de aula y ficha del museo elaborados por el alumnado. Recuperado de https://laaventuraderussell.blogspot.com/, https://drive.google.com/file/d/1cZU210MLXE05ryB-vrBq6saAhtqrllsu/view 
En nuestra primera parada, los pequeños grupos elaboraron un guiñol y una maqueta del puerto cordubense. La investigación dio paso a una salida por el entorno del río Guadalquivir y una visita a la zona donde se estableció el puerto de Corduba, para que conozcan su situación estratégica y las relaciones comerciales que tuvo con otras provincias del Imperio romano. Para documentar previamente a los/as alumnos/as, le presentamos un texto que narra la importancia que tuvo esta colonia por sus exportaciones de vino, aceite, trigo, plomo y plata, y para que también puedan conocer el sistema monetario de los romanos. Posteriormente, iniciamos un breve debate para que se fijen en la ubicación del espacio cultual de los Altos de Santa Ana de Córdoba, puedan reflexionar porqué esta plaza se destinó al culto público y diferencien las partes que componen su distribución original. Para la investigación de este espacio sacro consultaron a través de diversos enlaces web y descubrieron cómo se decoraba, a qué magistrados se representaban y qué imágenes religiosas aparecían en su entorno. La elaboración de un puzle hizo que a través de esta investigación los/as estudiantes llegaran a conclusiones de manera grupal, con el fin de canalizar sus respuestas y plasmarlas en el blog de aula en el que recogieron los edificios en los que se daba culto.

Para asomarse a la vida cotidiana dedicamos nuestro primer taller a los cultos públicos que se desarrollaron en la ciudad. Nos visitó un especialista (un arqueólogo) que explicó cómo se vivía en la sociedad de esa época, qué costumbres tenían, cómo eran las creencias en el ámbito público, y cómo se decoraba el foro con estatuas. Durante la explicación de esta parada, los/as alumnos/as trazaron en el suelo dos ejes que se cortaban para ubicar el cardo y el decumano y poder situar el foro romano. Del mismo modo, narraron con una escenificación cómo se representaban a los ciudadanos y a las ciudadanas más destacados en la plaza principal de una colonia romana.

Por lo que respecta a las ceremonias funerarias, el carácter religioso de estas liturgias se materializó con la representación de un ritual. Aprovechamos los Mausoleos de Puerta Gallegos y su ubicación fuera de la muralla para conocer los pasos que tenía esta liturgia y el cortejo fúnebre, los personajes que formaban parte de ella y las dimensiones que tenían los monumentos donde se enterraban a los fallecidos. Asimismo, aprovechamos los restos arqueológicos del anfiteatro romano que se encuentran in situ, formando parte del espacio urbano que ocupa el Rectorado de la Universidad de Córdoba. Aunque el edificio de espectáculos no se encuentra visible por su deterioro, los/as alumnos/as pudieron recrear un combate gladiatorio en un parque anejo al yacimiento. Utilizamos la cercanía de este parque para delimitar en el pavimento las partes del anfiteatro, su graderío y el lugar que ocupa cada escalafón social: desde magistrados de la colonia, hasta mujeres, libertos y esclavos (Figuras 4 y 5).

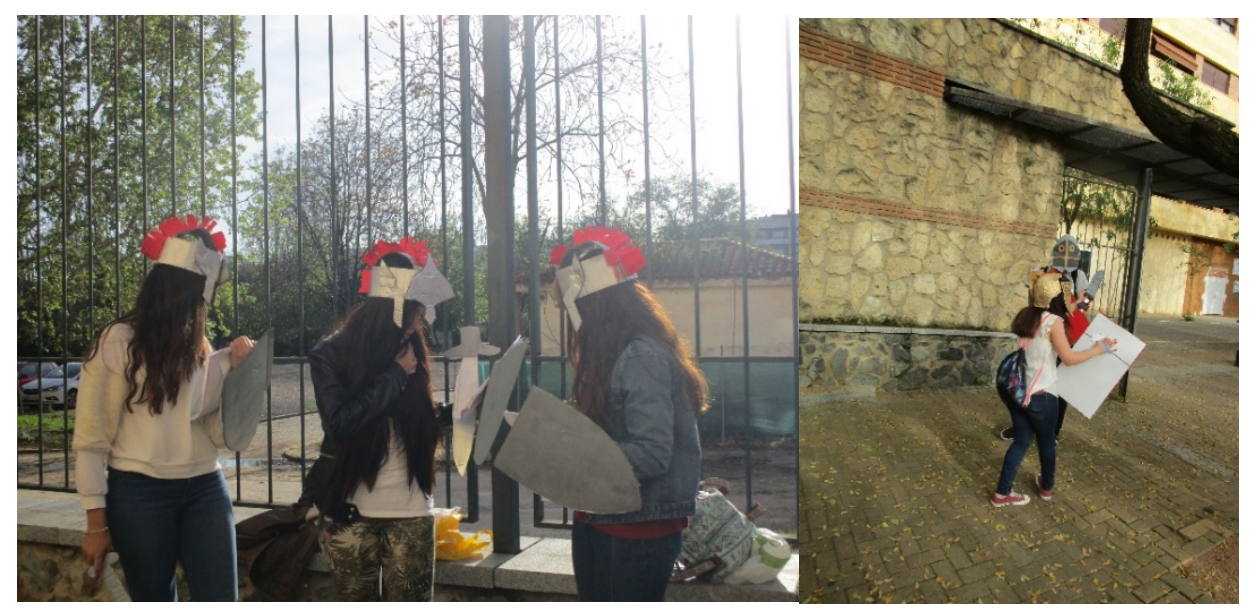

Figuras 4-5. Alumnos/as exponiendo en las distintas paradas del itinerario. Recreación de juegos en el anfiteatro romano. 
En cuanto a la visita al Museo Arqueológico de Córdoba, aquí los diferentes grupos trabajaron las mismas temáticas que en la salida para complementar los conocimientos que habían adquirido con las investigaciones previas. Estas investigaciones y la toma de contacto con la cultura material del museo sirvió para confeccionar un cuaderno didáctico con actividades adaptadas a los/as discentes de segundo y tercer ciclo de Educación Primaria. Las actividades que tuvieron varias fases se elaboraron de manera previa a la visita al museo, durante toda la visita y en una sesión final para mostrar cómo se vivía en tiempos de los romanos (Figuras 6, 7 y 8).

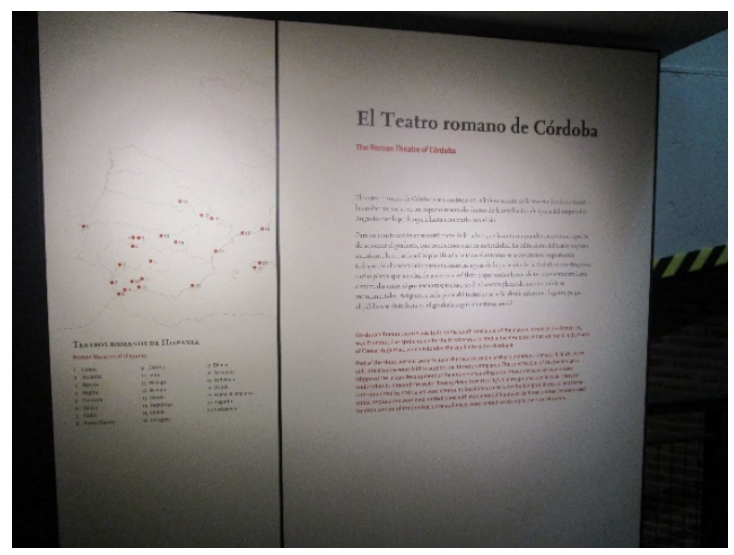

Figura 6. Panel informativo del Museo Arqueológico de Córdoba para contextualizar los restos in situ del teatro romano de Corduba.

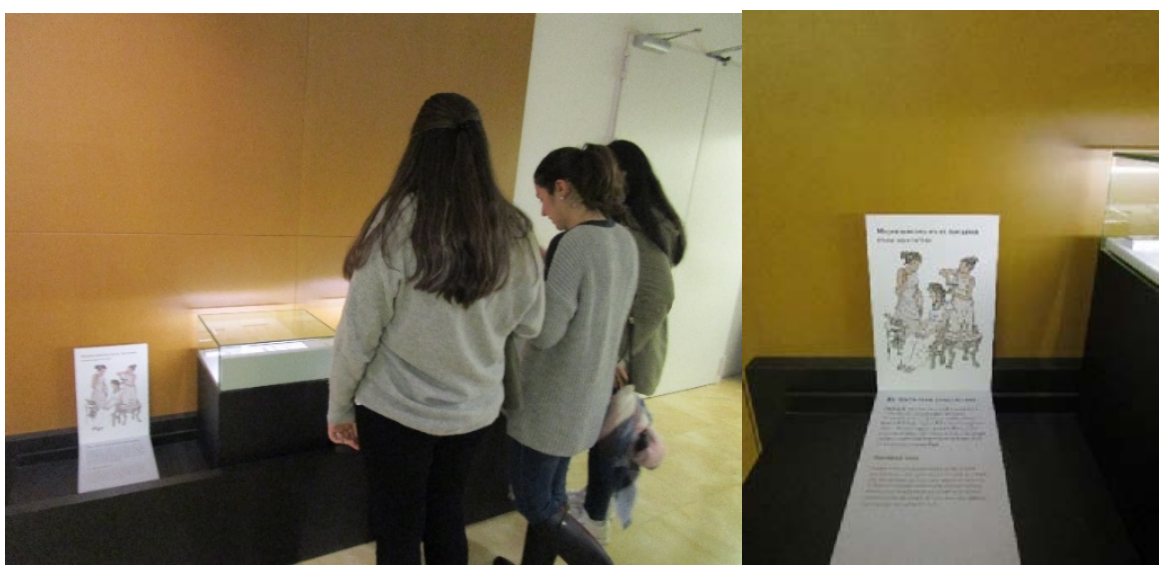

Figuras 7-8. Actividad coeducativa de arqueología de género y panel explicativo en la visita al Museo Arqueológico de Córdoba.

\section{RESULTADOS}

Para su formación académica y personal ha sido significativa esta manera de trabajar cooperativamente, ya que hemos podido reforzar la investigación, la presentación escrita y oral, la selección de contenidos y su posterior análisis, el trabajo individual y grupal, las estrategias para elaborar informes, y la utilización de las tecnologías de la información. Al respecto, Payà, Gros, Piqué \& Rubio (2018, p.443) afirman: "La conexión entre el conocimiento teórico y la práctica no se produce de forma espontánea, es necesario promover y dotar de instrumentos a los estudiantes para adquirir competencias profesionales adecuadas". Todo este proceso educativo se encuentra recogido en los indicadores de evaluación de las áreas mencionadas. 
El proyecto en sí mismo potencia una nueva forma de evaluar, más allá de los métodos tradicionales de enseñanza, con el objetivo de que los/as estudiantes elaboren una propuesta mediante la resolución de una serie de tareas centradas en el conocimiento de nuestra ciudad en época romana. Del mismo modo, la evaluación se cuantificó con un cuestionario de corte cuantitativo sobre diversas preguntas que hicieron que los/as alumnos/as reflexionaran sobre lo que habían aprendido y que habían aprendido a hacer (Seva, Soriano \& Vera, 2010). Para ello respondieron a diez ítems y una escala tipo Likert con cinco niveles que van desde el 1 que equivale a "de acuerdo" hasta el 5 "muy de acuerdo". La recogida de datos se vinculó con las cuestiones identitarias patrimoniales y sus impresiones sobre los talleres, la metodología, el itinerario y los interrogantes que se plantearon al comienzo de la experiencia didáctica (tabla 1).

Tabla 1.

Cuestionario para la recogida de información de la propuesta didáctica.

\begin{tabular}{|c|c|c|c|c|c|}
\hline \multirow{2}{*}{$\begin{array}{l}\text { Indicadores } \\
\text { 1) El patrimonio arqueológico debe ser cuidado y } \\
\text { conservado. }\end{array}$} & 1 & 2 & 3 & 4 & 5 \\
\hline & & & & & \\
\hline \multicolumn{6}{|l|}{$\begin{array}{l}\text { 2) En la asignatura de Didáctica de las Ciencias } \\
\text { Sociales realizamos salidas arqueológicas. }\end{array}$} \\
\hline \multicolumn{6}{|l|}{$\begin{array}{l}\text { 3) En la enseñanza de las Ciencias Sociales es } \\
\text { fundamental hacer actividades de difusión } \\
\text { patrimoniales. }\end{array}$} \\
\hline \multicolumn{6}{|l|}{$\begin{array}{l}\text { 4) Los recursos didácticos pueden ser una } \\
\text { estrategia para conocer mejor el patrimonio } \\
\text { histórico y arqueológico. }\end{array}$} \\
\hline $\begin{array}{l}\text { 5) Desde mi experiencia docente, en el Prácticum } \\
\text { de Educación Primaria, es provechoso transmitir } \\
\text { conocimientos histórico-arqueológicos en la } \\
\text { escuela. }\end{array}$ & & & & & \\
\hline
\end{tabular}

6) Para que la enseñanza sea activa debo implicarse directamente en su entorno cultural y patrimonial.

7) Los proyectos educativos en la universidad favorecen el conocimiento del patrimonio cultural.

8) Las experiencias didácticas deben ser compartidas por el equipo docente para que la enseñanza sea más enriquecedora e interdisciplinar.

9) Es necesaria la implementación de las competencias educativas a través del pensamiento histórico y arqueológico.

10) Resulta enriquecedor desarrollar hábitos de cooperación y de trabajo en equipo.

Fuente: elaboración propia.

\section{CONCLUSIONES}

A lo largo de toda esta trayectoria didáctica se han podido identificar fortalezas y dificultades del trabajo por proyectos para investigar la Corduba romana. Por un lado, las fortalezas permitieron mantener a un alumnado motivado y unos niveles competenciales adecuados (Villa \& Poblete, 2007), con los que el grupo diseñó y planificó su aprendizaje, y al mismo tiempo mejoró su interacción entre los/as compañeros/as y el fomento de la inclusión. Sin embargo, las limitaciones se centraron en la desconfianza del grupo hacia la metodología utilizada, lo que provoca la falta de motivación del alumnado al emprender un proyecto del que desconoce la temática y las 
fases de un trabajo esencialmente investigativo al que no está acostumbrado en otras asignaturas del grado. Además, es necesario señalar que la propuesta metodológica ha supuesto una opción a los métodos tradicionales de enseñanza y el interés del alumnado con la investigación-acción (Latorre, 2003). De hecho, esta experiencia educativa muestra una significativa mejoría en el aprendizaje de los conocimientos históricos, el interés por la investigación histórica y el trabajo grupal y, sobre todo, la implicación del alumnado del Grado de Educación Primaria para identificar las huellas del pasado romano que aún permanecen en Córdoba.

A este respecto Levy (2017) apunta que los estudiantes de la escuela construyen sus narrativas con eventos que les son familiares, lo que facilita un pensamiento histórico comprometido con sus experiencias patrimoniales. Se trata, en definitiva, de pensar la historia y adquirir los conocimientos adecuados para investigar cómo se conservan hoy día las culturas del pasado. En este sentido, los interrogantes de esta investigación se centraron en evaluar las percepciones del alumnado universitario con los métodos históricos que se implementan en sus clases y la implicación que tienen con el patrimonio arqueológico más cercano, material e inmaterial. Por último, Navarro, González, López \& Contreras (2019, p. 522), nos dicen: "los alumnos deben evaluar el proyecto realizado y determinar en qué grado su trabajo ha posibilitado la consecución de la meta común, o lo que es lo mismo, cuál ha sido el nivel de aprendizaje adquirido".

\section{REFERENCIAS}

Alonso, A. (2018). Aprendizaje Basado en Proyectos para el desarrollo de la Competencia Digital Docente en la Formación Inicial del Profesorado. Relatec: Revista Latinoamericana de Tecnología Educativa, 17(1), 9-24. DOI: https://doi.org/10.17398/1695-288X.17.1.9

Ballart, J. (2002). El patrimonio histórico y arqueológico: valor y uso. Barcelona: Ariel.

Cebrián, M. (coord.) (2003). Enseñanza virtual para la innovación universitaria. Madrid: Narcea.

Cozar, R., \& Sáez, J. M. (2017). Realidad aumentada, proyectos en el aula de primaria: experiencias y casos en Ciencias Sociales. EDMETIC, 6(1),165-180. DOI: https://doi.org/10.21071/edmetic.v6i1.5813

Cuenca, J. M., Estepa, J., \& Martín, M. J. (2011). El patrimonio cultural en la educación reglada. Revista de Patrimonio cultural de España. Patrimonio y Educación, 5, 45-57.

Durán, J. F. (2011). La contribución del Edublog como estrategia didáctica. Electronic Journal of Research in Educational Psychology, 9(1), 331-356.

García-Valcárcel, A., \& Basilotta, V. (2017). Aprendizaje Basado en Proyectos (ABP): evaluación desde la perspectiva de alumnos de Educación Primaria. Revista de Investigación Educativa, 35(1), 113-131. DOI: https://doi.org/10.6018/rie.35.1.246811

Hernández, A. M., \& Guillén, R. (2017). La educación patrimonial en los manuales escolares de Educación Primaria: un recorrido desde LOGSE hasta LOMCE. Didáctica de las Ciencias Experimentales y Sociales, 32(2), 25-49. 
Kolmos, A. (2004). Estrategias para desarrollar currículos basados en la formulación de problemas y organizados en base a proyectos. Educar, 33, 77-96. DOI: https://doi.org/10.5565/rev/educar.262

Lata, S., \& Castro, M. (2016). El Aprendizaje Cooperativo, un camino hacia la inclusión educativa. Revista Complutense de Educación, 27(3), 1085-1101. DOI: https://doi.org/10.5209/rev_RCED.2016.v27.n3.47441

Latorre, A. (2003). Investigación-acción. Conocer y cambiar la práctica educativa. Barcelona: Graó.

Latorre, A., Rincón, D., \& Arnal, J. (2005). Bases metodológicas de la investigación educativa. Barcelona.

Levy, S. (2017). How Students Navigate the Construction of Heritage Narratives. Theory \& Research in Social Education, 45(2), 157-188.

López, R., Miralles, P., Prats, J. y Gómez, C. (2017). Enseñanza de la historia y competencias básicas. Barcelona: Graó.

Martín, M., \& Cuenca, J. M. (2011). La enseñanza y el aprendizaje del patrimonio en los museos: la perspectiva de los gestores. Revista de Psicodidáctica, 6(1), 99-122.

Molina, M. P. (2018a). La educación patrimonial en la didáctica de las ciencias sociales en primaria. Contextos Educativos. Revista de Educación, 22, 199-213. DOI: http://dx.doi.org/10.18172/con.3087

Molina, M. P. (2018b). Aprendizaje basado en proyectos (ABP) para la enseñanza de la Historia. En A. I. Ponce y J. Ortuño (Eds.), Pensando el patrimonio: Usos y recursos en el ámbito educativo (pp. 127-138). Murcia: Universidad de Murcia.

Moliner, O., \& Sánchez-Tarazaga, L. (2015). PBL o Aprendizaje Basado en Proyectos: una experiencia de coordinación y enseñanza auténtica en la universidad. Quaderns digitals: Revista de Nuevas Tecnologías y Sociedad, 81, 116-133.

Navarro, I., González, C., \& Botella, P. (2015). Aprendizaje basado en proyectos: Diferencias percibidas en la adquisición de competencias por el alumnado universitario. Revista de Psicología y Educación, 10(1), 55-76.

Navarro, I., González, C., López, B., \& Contreras, A. (2019). Aprendizaje cooperativo basado en proyectos y entornos virtuales para la formación de futuros maestros. Educar, 55(2), 519-541. DOI: https://doi.org/10.5565/rev/educar.935

Palma, A. (2013). Una propuesta didáctica para la enseñanza-aprendizaje del tiempo histórico y el espacio geográfico en el Grado de Maestro en Educación Primaria. Clío: History and History Teaching, 39, 1-16. Recuperado de: dialnet.unirioja.es/servlet/articulo?codigo $=4532553$.

Payà, M., Gros, B., Piqué, B. y Rubio, L. (2018). Co-creación y validación de instrumentos para la integración del conocimiento experiencial en la formación de maestros. Profesorado. Revista de Currículum y Formación del Profesorado, 22(1), 441-460.

Pérez, A. (2010). Aprender a educar. Nuevos desafíos para la formación de docentes. Revista Interuniversitaria de Formación del Profesorado, 68(24), 37-60. 
Perrenoud, P. (2004). Diez nuevas competencias para enseñar. Barcelona: Graó.

Prats, J. (2008). Memoria histórica versus historia enseñada. Íber. Didáctica de las Ciencias Sociales, Geografía e Historia, 55, 5-8.

Pujolàs, P. (2008). El aprendizaje cooperativo. Barcelona: Graó.

Rekalde, I., \& García, J. (2015). El aprendizaje basado en proyectos: un constante desafío. Innovación educativa, 219-234. DOI: http://dx.doi.org/10.15304/ie.25.2304

Riera, G. (2011). El aprendizaje cooperativo como metodología clave para dar respuesta a la diversidad del alumnado desde un enfoque inclusivo. Revista Latinoamericana de Educación Inclusiva, 5(2), 113-149.

Rüsen, J. (2005). History: Narration, Interpretation, Orientation. New York: Berghahn Books.

Saiz, J., \& Gómez, C. J. (2016). Investigar el pensamiento histórico y narrativo de la formación del profesorado: fundamentos teóricos y metodológicos. Revista Electrónica Interuniversitaria de Formación del Profesorado, 19(1), 175-190. DOI: http://dx.doi.org/10.6018/reifop.19.1.206701

Salinas, J., De Benito, B., \& Lizana, A. (2014). Competencias docentes para los nuevos escenarios de aprendizaje. Revista Interuniversitaria de Formación del Profesorado, 28(1), 145-163.

Seva, F., Soriano, M. C., \& Vera, M. I. (2010). La práctica docente en las Ciencias Sociales: un análisis cuantitativo. En R. M. Ávila, M. P. Rivero y P. L. Domínguez, (Coords.), Metodología de Investigación en Didáctica de las Ciencias Sociales (pp.175-183). Zaragoza.

Vergara, J. J. (2016). Aprendo porque quiero. El aprendizaje basado en proyectos $(A B P)$, paso a paso. Madrid: SM.

Villa, A., \& Poblete, M. (2007). Aprendizaje basado en competencias. Una propuesta para la evaluación de las competencias genéricas. Bilbao: Universidad de Deusto. 